\title{
Novel fiber optic accelerometer system using geometric moiré fringe
}

\author{
Maria Q. Feng, Dae-Hyun Kim* \\ Department of Civil and Environmental Engineering, University of California, Irvine, CA 92697-2175, USA \\ Received 26 October 2005; received in revised form 13 December 2005; accepted 30 December 2005 \\ Available online 14 February 2006
}

\begin{abstract}
This paper presents an innovative fiber optic accelerometer system for monitoring vibration of large-size structures. The system is composed of one (or multiple) sensor head and a control unit for driving the sensor and processing sensor data. The sensing mechanism is based on a novel integration of the moiré fringe phenomenon with fiber optics, resulting in accurate and reliable measurement. A prototype fiber optic accelerometer system has been successfully developed, including a sensor head, a low-cost control unit and a software package with a unique algorithm for processing the moiré fringe signals into accelerations with a high resolution. Finally, free vibration and shaking table tests were performed to identify the dynamic characteristics and demonstrate the high performance of the sensor system developed in this study.
\end{abstract}

(C) 2006 Elsevier B.V. All rights reserved.

Keywords: Fiber optic; Accelerometer; Moiré fringe; Monitoring; Civil structure

\section{Introduction}

Our nation's civil infrastructure (including bridges, electrical power grids, dames, pipelines, and school buildings) received a D grade from the American Society of Civil Engineers [1], raising serious concerns about public safety and associated substantial economic loss. Sensor-based monitoring can establish more scientific prioritization for structural repair and rehabilitation, provide early warning before catastrophic structural failure caused by natural or man-made disasters, and permit real-time post-disaster damage assessment. However, our civil infrastructure has not benefited from sensor-based monitoring due to the unavailability of cost-effective and suitable sensors. Civil infrastructure systems place unique demands on sensors. Besides accuracy, sensors and their cables are expected to be reliable, low in cost, light in weight, small in size, resistant to EM interference, and long in service life. They are required to withstand harsh environments, be moisture-, explosion-, lightning-proof, and corrosion-resistant. Civil engineering structures are usually very large, demanding easy cabling of the sensors. It is very difficult, if not impossible, for the currently available electric-type sensors to satisfy these demanding requirements. For instance,

\footnotetext{
* Corresponding author. Tel.: +1 949351 8716; fax: +1 9498240187 E-mail address: daehk@uci.edu (D.-H. Kim).
}

these sensors use electric cables for signal transmission and power supply, which may act as large antennae picking up various kinds of noise, creating ground loops, and are susceptible to lightning strikes.

Emerging fiber optic sensor technologies have shown great potential to overcome the difficulties associated with the conventional sensors. They are immune to EM noise and electric shock and thus can be used in explosion-prone areas. Several kinds of fiber optic sensors have been developed over the last two decades to take advantage of these merits (e.g. [2,3]). There have also been many field applications of fiber optic sensors for health monitoring of civil engineering structures (e.g. [4-7]). However, very few optical fiber sensors, particularly dynamic sensors (e.g. accelerometers, dynamic strain gauges, and pressure sensors) have been successfully commercialized for monitoring civil engineering structures (e.g. [8-10]). Recent research suggests that structural integrity can be assessed by continuously monitoring structural vibration (e.g. [11-13]). This paper presents a new fiber optic accelerometer for monitoring large-size structures such as those in civil infrastructure systems. The sensing mechanism of the sensor head is based on a novel integration of the moiré fringe phenomenon with fiber optics. The moiré fringe phenomena have a long history of use in engineering measurement (e.g. [14,15]) and the proposed sensor takes the advantage of this well-established and reliable measurement technique by employing fiber optics in a novel way. 
In this study, a prototype fiber optic accelerometer system has been successfully developed that consists of a sensor head, a control unit for driving the sensor head and a signal processing unit equipped with a unique algorithm for processing the moiré fringe signals into directions and magnitudes of accelerations with a high resolution. Free vibration test was carried out to characterize the sensor's dynamic parameters such as the natural frequency and damping ratio. Finally, shaking table test demonstrated the high performance of the sensor system developed in this study.

\section{Principle of the sensing mechanism}

The proposed accelerometer head contains a pendulum that can be modeled as a single-degree-of-freedom dynamic system with a mass $m$, a spring stiffness $k$ and a damper $c$, as shown in Fig. 1. The equation of motion for the pendulum system is simply expressed as follows:

$m \ddot{x}+c \dot{x}+k x=-m \ddot{x}_{\mathrm{o}}$

where $x=x_{1}-x_{0}$, the relative displacement between the pendulum mass and the sensor casing (i.e. the pendulum support) and $\ddot{x}_{\mathrm{O}}$ is the acceleration imparted to the sensor (i.e. the "excitation acceleration") that is to be measured.

Eq. (1) can be rewritten in terms of the damping ratio $\zeta$ and the natural frequency of the pendulum $\omega_{\mathrm{n}}$ :

$\ddot{x}+2 \omega_{\mathrm{n}} \zeta \dot{x}+\omega_{\mathrm{n}}^{2} x=-\ddot{x}_{\mathrm{o}}$

where $\omega_{\mathrm{n}}=\sqrt{k / m}$ and $\zeta=c / 2 m \omega_{\mathrm{n}}$.

Assuming that $\ddot{x}_{\mathrm{o}}=A_{\text {excite }} \mathrm{e}^{\mathrm{i} \omega t}$, where $\omega$ is the frequency of the excitation acceleration, then the steady state response should be $x=D_{\text {response }} \mathrm{e}^{\mathrm{i} \omega t}$. In theory, the ratio of $D_{\text {response }}$ to $A_{\text {excite }}$ should satisfy the following relation:

$\frac{D_{\text {response }}}{A_{\text {excite }}}=\frac{-1}{\omega_{\mathrm{n}}^{2}-\omega^{2}+2 \mathrm{i} \omega_{\mathrm{n}} \omega \zeta}$

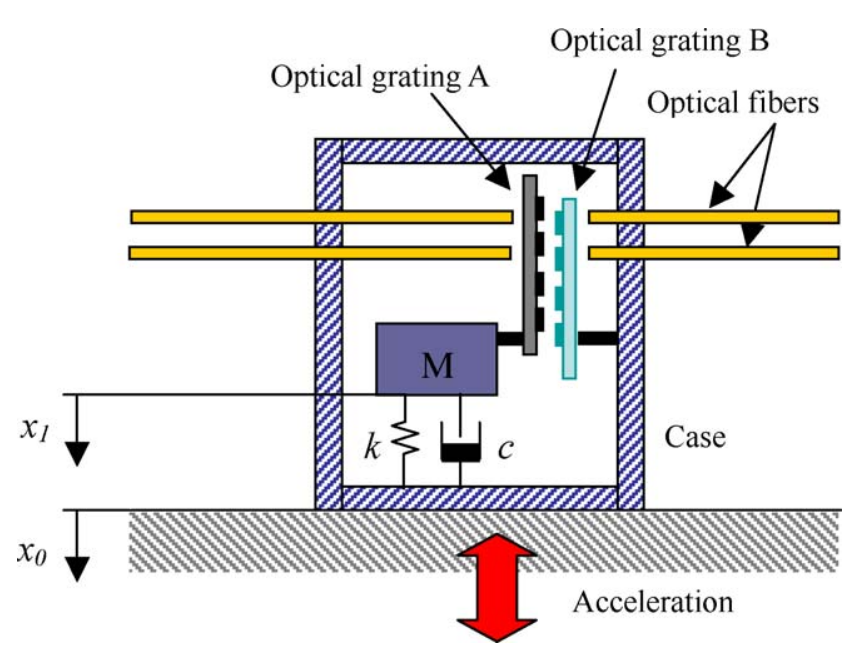

Fig. 1. Conceptual design of moiré fringe-based fiber optic accelerometer.
Thus, if one sets $r=\omega / \omega_{\mathrm{n}}$, then the deformation response factor, $R$ can be expressed as follows:

$R=\left|\frac{D_{\text {response }}}{A_{\text {excite }}}\right| \omega_{\mathrm{n}}^{2}=\frac{1}{\sqrt{\left(1-r^{2}\right)^{2}+(2 r \zeta)^{2}}}$

where $r=\omega / \omega_{\mathrm{n}}$.

One can carefully design a system with a pendulum of mass $m$ and spring stiffness $k$ such that the natural frequency $\omega_{\mathrm{n}}$ is much larger than $\omega$. As $\omega_{\mathrm{n}}$ increases, the ratio $r$ approaches zero, and in turn the deformation response factor, $R$ approaches unity. In this condition, Eq. (4) demonstrates that the relative displacement, $x$ is directly proportional to the excitation acceleration, $\ddot{x}_{0}$. Consequently, one can derive the acceleration of the sensor simply by gauging the relative displacement, $x$ between the pendulum mass and the sensor casing. In this paper, the proposed fiber optic accelerometer system applies a moiré fringe technique via gratings and optical fibers to reliably measure the relative displacement, $x$.

As shown in Fig. 1, the fiber optic accelerometer contains a pair of parallel optical grating panels, one fixed to the mass of the pendulum and the other to the sensor casing. As a result, the relative displacement of the two optical gratings is the same as the displacement between the mass and the sensor casing.

Because one optical grating is fixed to the mass while the other is fixed to the sensor casing, the relative displacement of the two optical gratings is the same as the displacement between the mass and the sensor casing. When two optical gratings consisting of alternating parallel transparent and opaque strips (i.e. "rulings") are overlaid, light will either be transmitted (when the transparent regions coincide) or be obstructed (when they do not coincide). If the rulings on one grating are aligned at a small angle relative to those on the other, then the loci of their intersections will be visible as dark moiré fringes running approximately perpendicular to the rulings as shown in Fig. 2.

As the two parallel grating panels move with respect to each other in the $X$ direction, the moiré fringes will move in the $Y$ direction. The moiré fringes will shift by one pitch distance $D$ in the $Y$ direction when the two gratings shift in the $X$ direction by

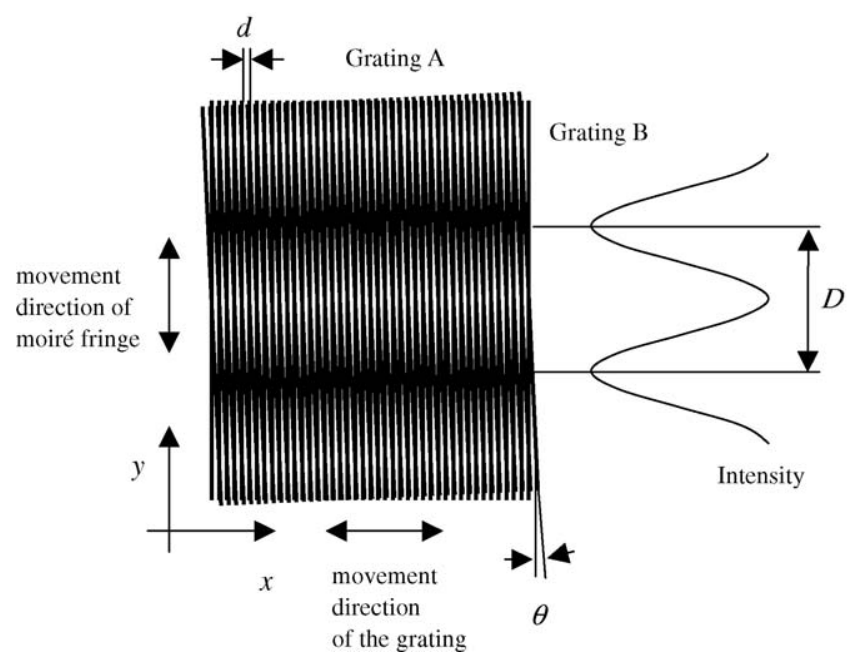

Fig. 2. Moiré fringes for displacement amplification. 
one grating pitch $d$. The moiré fringe pitch $D$ can be designed using the relation $D=d / \theta$, where $\theta$ is an angle subtended by the rulings of the two gratings. If one lets $\theta$ approach zero in the relation, the ratio $D / d$ (i.e. the "displacement amplification") can become very large. Consequently, if a small $\theta$ is chosen, one can considerably amplify a small grating movement $d$ into a large moiré fringe movement $D$. One would facilitate accurate displacement measurement by designing a small $\theta$ and thus a large $D$.

The relative displacement between the two gratings can be measured by tracking the moiré fringes as they pass through one point. However, observing the moiré fringes at only one point yields no information regarding the direction of their movement, which is necessary to determine the direction of the relative movement of the two gratings. Fortunately, one can determine the direction as well as the amplitude of the displacement by tracking the fringes at two points which are separated by a quarter of the fringe width $D$ across the fringe profile. In order to observe the moiré fringe at two different points, two pairs of optical fibers are placed perpendicular to the optical grating panels, as illustrated in Fig. 1.

\section{Signal processing algorithm}

The light intensity signals at the two observing points are sinusoidal function of displacement with a phase difference of $\pi / 4$ with respect to each other. When superposed on a DC component, the light intensities at these two observing points can be expressed as follows:

$s_{1}(t)=C_{1} \sin \left(\frac{2 \pi x(t)}{d}\right)+C_{2}$,
$s_{2}(t)=C_{3} \sin \left(\frac{2 \pi x(t)}{d}+\frac{\pi}{4}\right)+C_{4}=C_{3} \cos \left(\frac{2 \pi x(t)}{d}\right)+C_{4}$

where $C_{1}, C_{2}, C_{3}$ and $C_{4}$ are constants. The above expressions can be normalized as follows:

$\bar{s}_{1}(t)=\frac{s_{1}(t)-C_{2}}{C_{1}}=\sin \left(\frac{2 \pi x(t)}{d}\right)$,
$\bar{s}_{2}(t)=\frac{s_{2}(t)-C_{4}}{C_{3}}=\cos \left(\frac{2 \pi x(t)}{d}\right)$

The relative displacement $x(t)$ can be obtained by unwrapping the two signals as follows:

$x(t)=\frac{d}{2 \pi} \operatorname{unwrap}\left(\arctan \left(\frac{\bar{s}_{1}(t)}{\bar{s}_{2}(t)}\right)\right)$

Finally, Eq. (4) provides the scaling factor by which the measured relative displacement can be converted into the sensor's acceleration.

\section{Prototype fiber optic accelerometer system}

Based on the sensing mechanism presented above, a prototype fiber optic accelerometer, together with control and

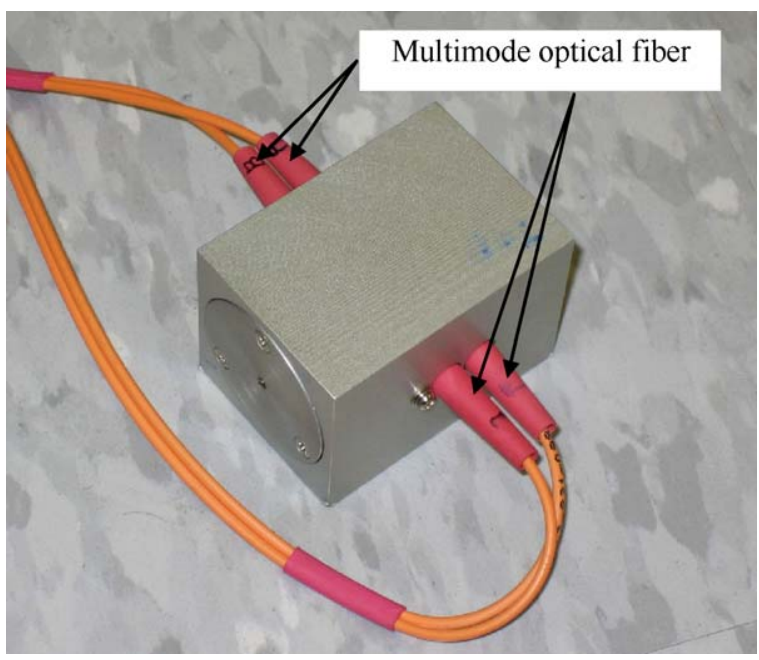

Fig. 3. Prototype fiber optic accelerometer head.

Table 1

Specifications of components used in fiber optic accelerometer

\begin{tabular}{ll}
\hline & Specifications \\
\hline Optical fiber & Graded index multimode fiber $(62.5 / 125 \mu \mathrm{m})$ \\
Lens & $f=1.81 \mathrm{~mm}$ GRIN lens \\
Grating pitch & $d$ (period) $=200 \mu \mathrm{m}$ \\
\hline
\end{tabular}

signal processing units, was successfully developed. Fig. 3 shows the prototype sensor head with a dimension of $33 \mathrm{~mm} \times 33 \mathrm{~mm} \times 48 \mathrm{~mm}$ and a weight of $150 \mathrm{~g}$.

The sensor head consists of a pendulum with a mass, a spring, and a damper, together with two glass gratings and two pairs of multimode optical fibers. In addition, a lens is applied at the tip of the each optical fiber to reduce the coupling loss between two optical fibers. The specifications of the optical fiber, the lens and the gratings are shown in Table 1 . The sensor head is linked to the control unit by the two optical fibers.

Fig. 4 shows the control unit developed in this study for driving eight sensor heads and processing the sensor signals. It provides the light source to each sensor head through the two optical fibers and detects the intensity variation of the light trans-

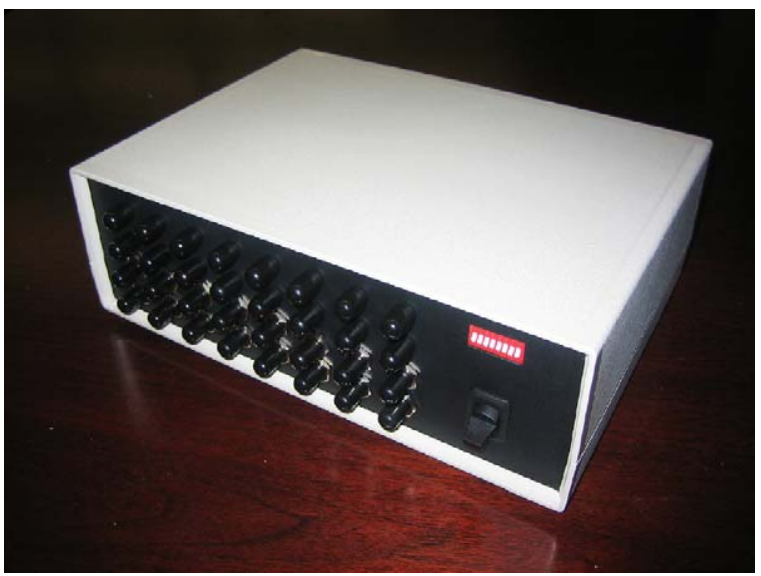

Fig. 4. Portable control unit for eight fiber optic sensors. 

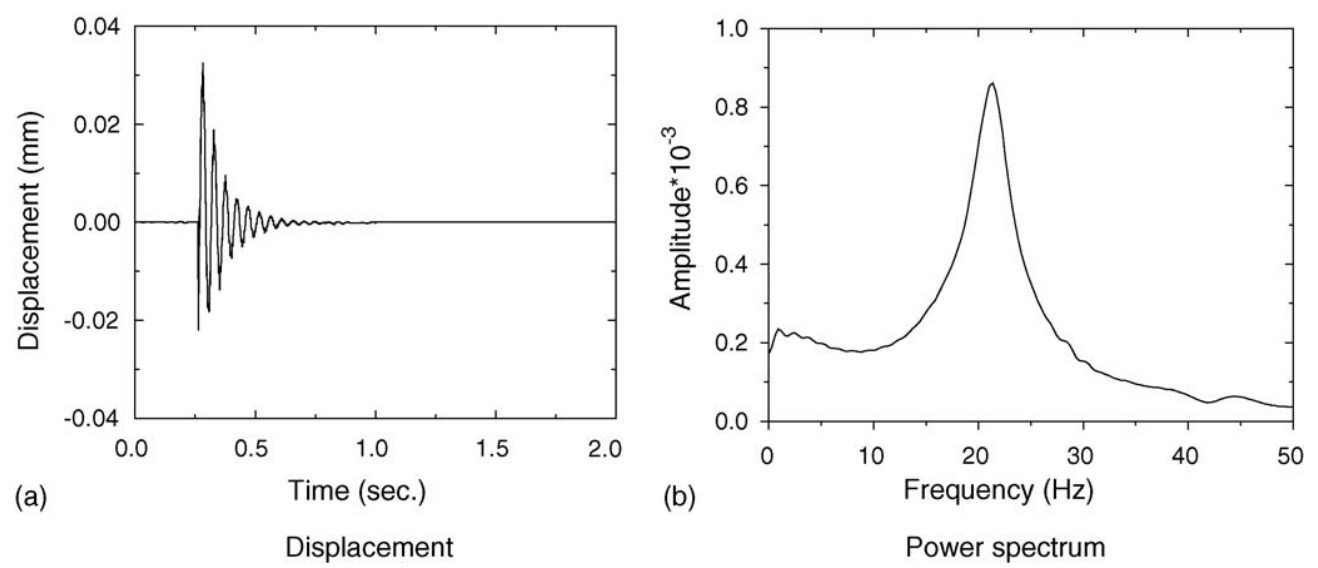

Fig. 5. Impulse response from free vibration test.

mitted through the two optical gratings. Totally 16 LEDs and 16 photo diodes are used in this unit. A rechargeable battery is included in the unit for portability. The control unit has a simple structure and the cost is much lower than many of the existing fiber optic sensor systems.

\section{Testing of fiber optic accelerometer}

Free vibration testing was first performed in order to identify the natural frequency and the damping ratio of the fiber optic accelerometer, which are needed for converting the raw optic signals to the acceleration as indicated in Eq. (4). Then, shaking table testing was carried out by mounting the fiber optic accelerometer together with a high-performance reference sensor on a small shaking table. The shaking table was excited by sinusoidal signals at selected frequencies covering a large range. Assuming the reference sensor accurately measures the sinusoidal excitation of the table, the deformation response curve can be plotted and compared with the theoretical one. The sensor performance can be evaluated based on the deformation response factor plots and the comparison with the reference sensor.

\subsection{Free vibration testing for parameter identification}

First free vibration of the pendulum inside the sensor head was induced by an impact hammer. The $90^{\circ}$ phase shifted raw optical signals at the two view points on the grating panels were recorded. The sampling rate for the optical signals was set to $5 \mathrm{kS} / \mathrm{s}$, and the time duration per each measurement was $2 \mathrm{~s}$. The free-decay relative displacement $x(t)$ was then calculated by Eq. (7). Fig. 5 shows the displacement time history and its power spectrum. From the time history $x(t)$ and its power spectrum, the natural frequency and damping ratio of the pendulum were identified as $f_{\mathrm{n}}=\omega_{\mathrm{n}} /(2 \pi)=21.4 \mathrm{~Hz}$ and $\zeta=9 \%$.

\subsection{Shaking table testing for performance evaluation}

The prototype fiber optic accelerometer and a highperformance reference sensor (Kinemetrics, FBA-11) were fixed to the shaking table (APS-Dynamics, model 113). The refer- ence sensor is an electric sensor equipped with a pendulum and a servo control circuit to achieve a large bandwidth (DC to $200 \mathrm{~Hz}$ ). A fixed-sine function generator was used to control the shaking table. The excitation frequencies were selected as $0.5 \mathrm{~Hz}, 1.0 \mathrm{~Hz}, 2.5 \mathrm{~Hz}, 5.0 \mathrm{~Hz}, 10.0 \mathrm{~Hz}, 15.0 \mathrm{~Hz}, 16.0 \mathrm{~Hz}$, $17.0 \mathrm{~Hz}, 18.0 \mathrm{~Hz}, 19.0 \mathrm{~Hz}, 20.0 \mathrm{~Hz}, 21.0 \mathrm{~Hz}, 21.5 \mathrm{~Hz}, 22.0 \mathrm{~Hz}$, $23.0 \mathrm{~Hz}, 24.0 \mathrm{~Hz}, 25.0 \mathrm{~Hz}, 30.0 \mathrm{~Hz}$, and $40.0 \mathrm{~Hz}$. Each test was performed five times in order to average out the errors associated with the signal acquisition and to evaluate the standard deviation of the data. Fig. 6 shows the experimental setup for the shaking table tests.

Fig. 7 shows a sequence of signals from raw signals being processed into the displacement.

The first two windows show the raw normalized signals and the third window the arctangent of the two normalized raw signals. The last window displays the displacement calculated by unwrapping the arctangent signal.

Fig. 8 plots the deformation response curve $R$ defined as ( $\left|D_{\text {response }} / A_{\text {excited }}\right| \omega_{\mathrm{n}}^{2}$ ) based on the testing results, in comparison with the theoretical curve expressed in Eq. (4) by using the identified natural frequency and damping ration.

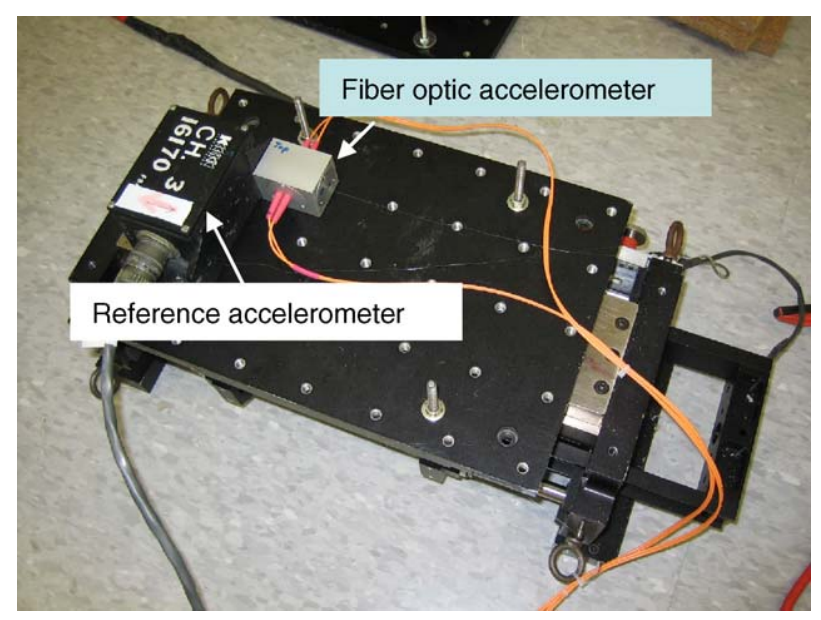

Fig. 6. Experimental setup for shaking table test. 


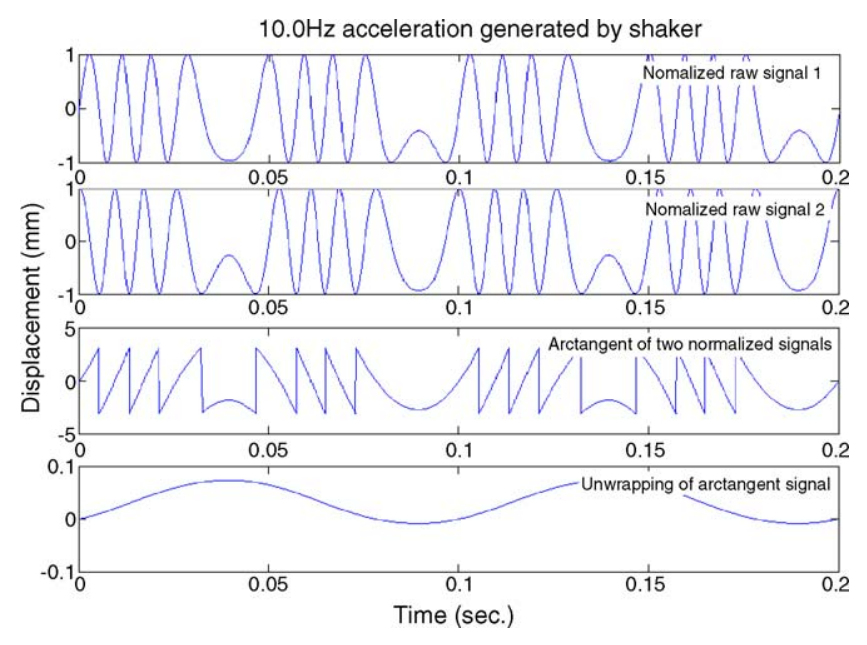

Fig. 7. Signal processing sequence.

Excellent agreement between the measured and the theoretical results are observed in Fig. 8, demonstrating the high accuracy of the fiber optic sensor system developed in this study. Also, a high repeatability of the sensor data was observed during the shaking table tests, indicating the high reliability of the sensor. Furthermore, the deformation response curve is flat up to $5 \mathrm{~Hz}$, indicating that the prototype accelerometer developed in this study can measure accelerations up to $5 \mathrm{~Hz}$ with less than $5 \%$ error. This sensor can be used for monitoring large-size civil engineering structures whose first few natural frequencies are below $5 \mathrm{~Hz}$. The dynamic bandwidth of fiber optic sensors can be further broadened by increasing the natural frequency of the pendulum system inside the sensor head and/or applying a software compensation filter.

Using the identified natural frequency, the displacements measured at the shaking table tests were converted into acceleration signals. Fig. 9 compares the accelerations at $0.5 \mathrm{~Hz}, 1 \mathrm{~Hz}$, and $5 \mathrm{~Hz}$ measured by the prototype fiber optic accelerometer with those by the high-performance reference sensor. Excellent agreement was observed.

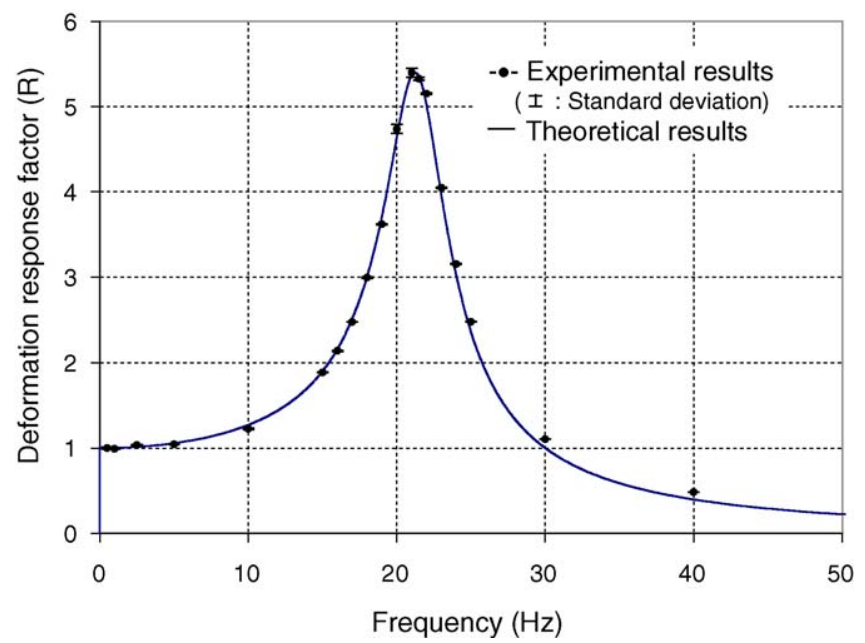

Fig. 8. Comparison of measured and theoretical deformation response factors.
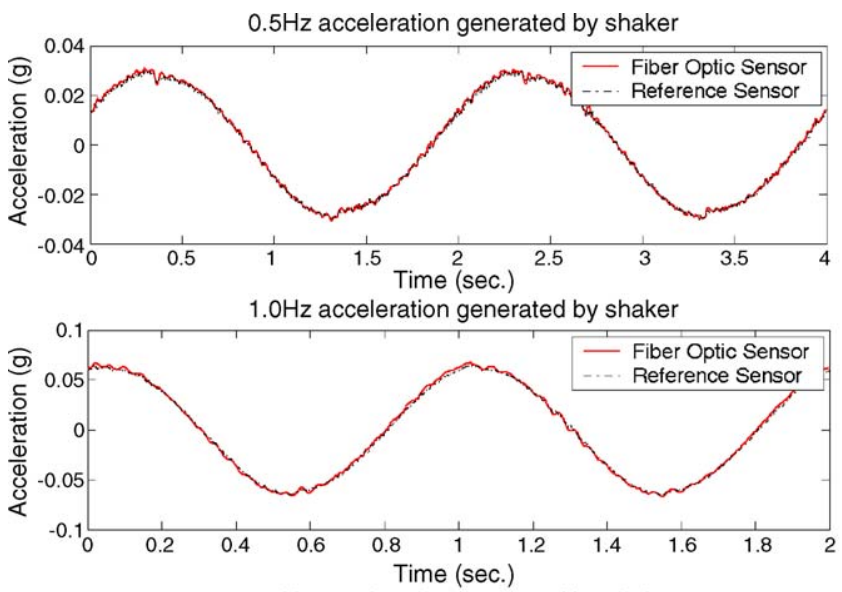

$5.0 \mathrm{~Hz}$ acceleration generated by shaker

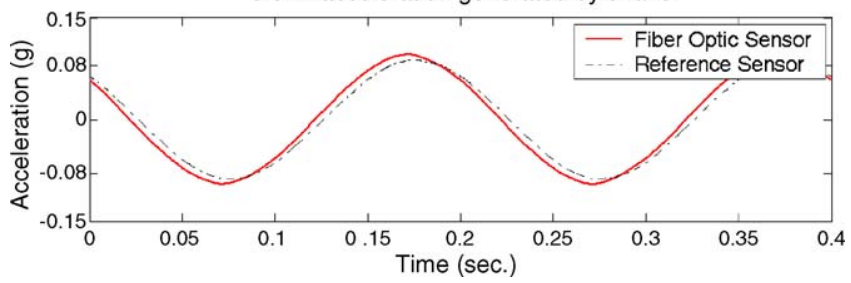

Fig. 9. Comparison of accelerations measured by fiber optic sensor and by reference.

\section{Concluding remarks}

This paper presents a novel fiber optic accelerometer system for monitoring dynamic response of large-size structures. The sensor head exploits the moiré fringe optical phenomenon and two pairs of optical fibers to accurately measure the displacement, and furthermore a pendulum to convert the displacement into acceleration. A prototype sensor system has been successfully developed, which consists of a sensor head and a control unit, equipped with a signal processing algorithm for processing the raw moire fringe signals into the direction and amplitude of the displacement and furthermore acceleration. The natural frequency and damping ratio of the prototype accelerometer were identified through free vibration testing. Finally, shaking table tests demonstrated the high accuracy and reliability of this unique fiber optic accelerometer system.

\section{Acknowledgements}

This work was supported by the National Science Foundation under award no. CMS-0329572 and the Korea Research Foundation Grant funded by Korea Government (MOEHRD, Basic Research Promotion Fund, D00276).

\section{References}

[1] American Society of Civil Engineers, ASCE, Infrastructure Report Card ASCE News 30 (3) (2005) 1-7.

[2] B. Lee, Review of the present status of optical fiber sensors, Opt. Fiber Technol. 9 (2003) 57-79.

[3] E. Udd, et al., in: E. Udd (Ed.), Fiber Optic Smart Structures, Wiley, New York, 1995. 
[4] F. Ansari, Fiber optic health monitoring of civil structures using long gage and acoustic sensors, Smart Mater. Struct. 14 (2005) S1-S7.

[5] H.N. Li, D.S. Li, G.B. Song, Recent applications of fiber optic sensors to health monitoring in civil engineering, Eng. Struct. 26 (2004) $1647-1657$.

[6] W. Moerman, et al., Measuring ground anchor forces of a Quay Wall with Bragg sensors, J. Struct. Eng. 131 (2) (2005) 322-328.

[7] R. Suresh, S.C. Tjin, N.Q. Ngo, Application of a new fiber Bragg grating based shear force sensor for monitoring civil structural components, Smart Mater. Struct. 14 (2005) 982-988.

[8] K. Kageyama, et al., Acoustic emission monitoring of a reinforced concrete structure by applying new fiber-optic sensors, Smart Mater. Struct. 14 (2005) S52-S59.

[9] D.H. Kim, et al., Damage detection of composite structures using a stabilized extrinsic Fabry-Perot interferometric sensor system, Smart Mater. Struct. 13 (3) (2004) 593-598.

[10] D.H. Kim, C.G. Kim, C.S. Hong, Simultaneous monitoring of crack signals and strain of composites using a stabilized EFPI sensor system, Key Eng. Mater. 270-273 (2004) 2146-2151.

[11] S.W. Doebling, C.R. Farrar, M.B. Prime, D.W. Shevitz, 1996. Damage Identification and Health Monitoring of Structural and Mechanical Systems from Changes in their Vibration Characteristics, A Literature Review, Los Alamos National Laboratory report LA-13070-MS.

[12] M.Q. Feng, E.Y. Bahng, Damage assessment of jacketed RC columns using vibration tests, J. Struct. Eng., ASC 125 (3) (1999) 265-271.
[13] M.Q. Feng, J.M. Kim, Identification of a dynamic system using ambient vibration measurements, J. Appl. Mech., ASME 65 (2) (1998) $1010-1023$

[14] S.P. Theocaris, Moiré Fringes in Strain Analysis, Pergamon Press, New York, 1969.

[15] D. Post, et al., High Sensitivity Moire, Springer-Verlag, New York, 1994.

\section{Biographies}

Dr. Dae-Hyun Kim has been working at University of California Irvine as a post-doctoral researcher since October 2004. He is currently developing a novel high-performance portable optical fiber accelerometer system for monitoring of civil infrastructures. His current research interests are optical sensor system, health monitoring, composite materials, structural analysis, etc. He received B.S. and M.S. degree from Department of Aerospace Engineering, Korea Advanced Institute of Science and Technology, in 1997 and 1999 respectively; Ph.D degree from Department of Mechanical Engineering, Korea Advanced Institute of Science and Technology, in 2004.

Dr. Feng is Professor in Department of Civil and Environmental Engineering at University of California Irvine. Her research interests are structural health monitoring, damage assessments, sensor developments, etc. She received $\mathrm{Ph} . \mathrm{D}$ degree from Department of Mechanical Engineering, University of Tokyo, in 1992. 